\title{
An analytical solution to calculate bulk mole fractions for any number of components in aerosol droplets after considering partitioning to a surface layer
}

\section{Topping}

National Centre for Atmospheric Science, NCAS, University of Leeds, UK

Centre for Atmospheric Science, University of Manchester, Manchester, UK

Received: 14 June 2010 - Published in Geosci. Model Dev. Discuss.: 13 July 2010

Revised: 14 October 2010 - Accepted: 21 October 2010 - Published: 4 November 2010

\begin{abstract}
Calculating the equilibrium composition of atmospheric aerosol particles, using all variations of Köhler theory, has largely assumed that the total solute concentrations define both the water activity and surface tension. Recently however, bulk to surface phase partitioning has been postulated as a process which significantly alters the predicted point of activation. In this paper, an analytical solution to calculate the removal of material from a bulk to a surface layer in aerosol particles has been derived using a well established and validated surface tension framework. The applicability to an unlimited number of components is possible via reliance on data from each binary system. Whilst assumptions regarding behaviour at the surface layer have been made to facilitate derivation, it is proposed that the framework presented can capture the overall impact of bulk-surface partitioning. Demonstrations of the equations for two and five component mixtures are given while comparisons are made with more detailed frameworks capable at modelling ternary systems at higher levels of complexity. Predictions made by the model across a range of surface active properties should be tested against measurements. Indeed, reccomendations are given for experimental validation and to assess sensitivities to accuracy and required level of complexity within large scale frameworks. Importantly, the computational efficiency of using the solution presented in this paper is roughly a factor of 20 less than a similar iterative approach, a comparison with highly coupled approaches not available beyond a 3 component system.
\end{abstract}

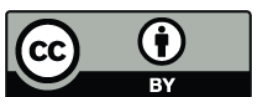

Correspondence to: D. Topping (david.topping@manchester.ac.uk)

\section{Introduction and rationale}

The process by which aerosol particles indirectly scatter radiation via activation to cloud droplets remains one of the largest uncertainties of climate change Halquist et al. (2009). The relationship between equilibrium size, chemical composition and ambient conditions is given by the Köhler equation.

$$
\frac{\mathrm{RH}}{100}=a_{\mathrm{w}} \exp \left(\frac{2 v_{\mathrm{w}} \sigma_{\mathrm{ws}}}{R T r_{\mathrm{drop}}}\right)
$$

where RH in the ambient relative humidity, $a_{\mathrm{w}}$ is the water activity (dimensionless), $v_{\mathrm{w}}$ the partial molar volume of water $\left(\mathrm{m}^{-3} \mathrm{~mol}^{-1}\right), \sigma_{\mathrm{ws}}$ the solution surface tension $\left(\mathrm{N} \mathrm{m}^{-1}\right)$, $R$ is the ideal gas constant $\left(8.314 \mathrm{~J} \mathrm{~K}^{-1} \mathrm{~mol}^{-1}\right)$ and $r_{\text {drop }}$ the radius of the droplet $(\mathrm{m})$. As discussed by Topping et al. (2007), calculating the equilibrium composition using all variations of Köhler theory has largely assumed that the total solute concentrations define both the water activity and surface tension. Recently however, bulk to surface phase partitioning has been postulated as a process which significantly alters the predicted point of activation (e.g. Sorjamaa et al., 2004; Laaksonen et al., 1999; Kokkola et al., 2006). Briefly, accounting for this process is based on the fact that different concentrations in a bulk and surface layer impact the number of moles of a compound defining both the raoult and kelin terms within the Köhler equation. However, in Gibbs surface thermodynamics the surface phase between the homogeneous liquid and vapour phase is taken to be infinitely thin, allowing an exact definition of the droplet radius (Sorjamaa et al., 2004). Any extra material which is related to the

Published by Copernicus Publications on behalf of the European Geosciences Union. 
change in surface tension is described using surface excess quantities which can be positive or negative:

$n_{\mathrm{it}}=n_{\mathrm{ib}}+n_{\text {is }}$

Where $n_{\mathrm{it}}$ is the total amount of component $i, n_{\mathrm{ib}}$ and $n_{\mathrm{is}}$ the bulk and surface phase concentrations respectively (mol). In order to solve these quantities, a solution to the Gibbs adsorption equation must be found. This relates the change in surface energy to changes in thermodynamic variables and excess quantities:

$\sum_{i} n_{\mathrm{ib}} d \mu_{i}+A_{\mathrm{drop}} d \sigma_{\mathrm{ws}}=0$

Where $\mu_{i}$ is the chemical potential of component $i\left(\mathrm{~J} \mathrm{~mol}^{-1}\right)$, usually re-written as $\mu_{i}+\mathrm{RT} \ln \left(a_{i}\right)$ where $\mu_{i}$ is the energy of formation of compound $i$ in the chosen reference state $\left(\mathrm{J} \mathrm{mol}^{-1}\right)$ and $a_{i}$ the activity of component $i$ (dimensionless). $A_{\text {drop }}$ is the surface area of the droplet $\left(\mathrm{m}^{2}\right)$ and $\sigma_{\mathrm{ws}}$ the surface tension $\left(\mathrm{N} \mathrm{m}^{-1}\right)$. The size of the droplet is important since as the surface to volume ratio increases then depletion of material from the bulk becomes important for thermodynamic properties. There have been methods presented to solve Eq. (3), thus Eq. (2) (and calculate ceoncentrations in the bulk and surface layer), for up to 3 component systems (two solutes and water; Sorjamaa et al., 2004). In essence, one has to derive relationships such that the system is no longer represented with a set of indeterminate equations. Sorjamaa et al. (2004) devised a numerical technique for binary organic/water systems and ternary inorganic/organic/water systems using relationships for activity coefficients and surface tension isotherms. To increase the number of components would require an, as yet, undetermined set of additional relationships or unjustified assumptions. In this study we present a methodology for such an extension relying on accurate and well established surface tension models, validated using bulk empirical data. These validated models enable an elegant analytical solution to Eq. (3) for any number of components to be derived. Using this approach, it is possible to use data derived from binary systems to describe a complex mixture.

\section{Derivation}

There are a number of surface tension models capable of treating multi-component systems as reviewed by Topping et al. (2007). To calculate the surface tension in the model presented by $\mathrm{Li}$ and $\mathrm{Lu}$ (2001), the authors combined the Gibbs dividing surface, the Langmuir adsorption equation and an appropriate model for calculating activity coefficients in mixed solutions to arrive at two different schemes. The langmuir adsorption isotherm is a relationship which describes the number of adsorbed molecules on a surface to the concentration above that surface. The model to replicate surface tension in binary systems is given by $(\mathrm{Li}$ and $\mathrm{Lu}, 2001)$ :

$\sigma_{\mathrm{ws}}=\sigma_{\mathrm{w}}+R T \Gamma_{i}^{\mathrm{wo}} \ln \left(\frac{1}{1+K_{i} a_{i}}\right)$

Where $\sigma_{\mathrm{ws}}$ is the surface tension of the mixture $\left(\mathrm{mN} \mathrm{m}^{-1}\right), \sigma_{\mathrm{w}}$ is the surface tension of pure water $\left(\mathrm{mN} \mathrm{m}^{-1}\right), \Gamma_{i}^{\mathrm{wo}}$ is the saturated surface excess of solute $i\left(\mathrm{mmol} \mathrm{m}^{-2}\right), K_{i}$ the adsorption equilibrium constant for solute $i$ (dimensionlesstabulated) and $a_{i}$ the activity of component $i$ (dimensionless). The reader is reminded that activity represents an effective concentration which relies on calculations of activity coefficients. Activity coefficients are related to the molecular forces taking place in a solution. The thermodynamics of a solution mixture however depend on the intermolecular forces that operate between molecules and are dependent on the nature of the solvent(s) and solutes. For more information the reader is referred to Topping et al. (2005) and references therein. As stated by $\mathrm{Li}$ and $\mathrm{Lu}$ (2001), the quantity $\Gamma_{i}^{\mathrm{wo}}$ is defined as the excess of $i$ in a unit surface area of the surface region over the moles which would be present in the bulk liquid phase containing the same number of moles of water as does the section of surface region. The superscript wo indicates that the dividing surface is chosen so that $\Gamma_{\mathrm{w}}=0$, or $n_{\mathrm{w}}^{s}=0$. Re-expressing this in relation to the Gibbs adsorption Eq. (3) and choosing $\Gamma_{\mathrm{w}}=0$, Eq. (3) reduces to Eq. (5):

$-d \sigma_{\mathrm{ws}}=\Gamma_{i}^{\mathrm{wo}} R T d \ln \left(a_{i}\right)$

For a binary system, It is then possible to solve the set of equations for a given dry and wet particle size (thus volume), pure component properties and surface tension parameters as described by Sorjamaa et al. (2004).

For multi-component systems, $\mathrm{Li}$ and $\mathrm{Lu}$ (2001) proposed two surface tension methods. These semi-empirical methods have been reviewed by Topping et al. (2007) and found to reproduce experimental data more accurately than entirely predictive methods. Thus we use the empirical validation of these methods against bulk data as a driving force for their use. This ultimately enables us to arrive at a simple solution for bulk/surface partitioning in a mixture of any number of components. Firstly, $\mathrm{Li}$ and $\mathrm{Lu}$ (2001) proposed that the adsorption behaviour of solute $i$ still followed the Langmuir gas-solid adsorption model, applied previously, in a mixed solution. It assumes that there is no interaction, nor competing adsorption, from the other solutes. In other words, it assumes that the relationship defining the surface excess used in a binary system still holds in a mixture. The expression for the multi-component surface tension is given as Eq. (6):

$\sigma_{\mathrm{ws}}=\sigma_{\mathrm{w}}+R T \sum_{i=1}^{k} \Gamma_{i}^{\mathrm{wo}} \ln \left(\frac{1}{1+K_{i} a_{i}}\right)$

As discussed by Fainerman et al. (2002), most authors have proposed procedures or models to predict the adsorption behaviour for a surfactant mixture from the known surface characteristics of the single compound. This naturally 
requires detailed experimental studies of surface tensions of individual solutions, and often, additional parameters that account for the mutual influence of solutes. This imposes certain restrictions on the capability of such approaches (Fainerman et al., 2002). Fainerman et al. (2002) derived a general but simple approximate expression for the surface tension of a surfactant mixture that allows estimation of the characteristics of a mixed solution, without any detailed analysis of the behaviour of the individual solutions and/or any account for specific interactions between the mixed species. It has been shown that it is possible to predict the surface tension of a mixed solution of two (or even " $n$ ") surfactants of different natures from the surface tensions for the individual solutions using this scheme. Fainerman et al. (2001) state this validity is ascribed to the fact that many particular features of adsorption process in mixed components (surface layer non-ideality, capability to reorient at the surface layer etc) are accounted for automatically because the surface tensions of the individual solutions are used. This is the same ethos used in this study. Solving the Gibbs adsorption equation for each binary system relies on the same assumption employed by Li et al. (1998) who defined the dividing surface as the equimolar surface with respect to water. Whilst this is different to the approach used by Laaksonen et al. (1999), it is unlikely that there will be any recognisable differences at droplet sizes larger than $10 \mathrm{~nm}$ (Sorjamaa et al., 2004). When Eq. (4) is used to describe the surface tension of binary systems in the Fainerman et al. (2002) mixing rule then predictions for multicomponent organic aqueous systems are identical to the full $\mathrm{Li}$ and $\mathrm{Lu}$ (2001) thermodynamic model shown in Eq. (6) (Topping et al., 2007). The Gibbs adsorption isotherm can now be used to describe the behaviour of each component in a mixture as it would pertain in a binary system. With an expression for the multicomponent surface tension we can now proceed to derive a general expression for altered mole fractions in the solution due to partitioning. Re-expressing the logarithm in Eq. (6) we get the following:

$\sigma_{\mathrm{ws}}=\sigma_{\mathrm{w}}-R T \sum_{i=1}^{k} \Gamma_{i}^{\mathrm{wo}} \ln \left(1+K_{i} a_{i}\right)$

To derive the generalised expression, we now need to convert the Gibbs adsorption isotherm into a complete differential (Eq. 5). Assuming chemical equilibrium between bulk and surface layer:

$$
\begin{array}{r}
n_{\mathrm{is}} d\left(R T \ln \left(x_{i \mathrm{~B}} \gamma_{i \mathrm{~B}}\right)\right)+A d \sigma_{\mathrm{ws}}=0 \\
n_{\mathrm{is}} d \ln \left(x_{i \mathrm{~B}} \gamma_{i \mathrm{~B}}\right)+A \frac{d \sigma}{\mathrm{RT}}=0 \\
n_{\mathrm{is}} \frac{d \ln \left(x_{i \mathrm{~B}} \gamma_{i \mathrm{~B}}\right)}{d x_{i \mathrm{~B}}}+\frac{A}{R T} \frac{d \sigma}{d x_{i \mathrm{~B}}}=0
\end{array}
$$

Note that we assume the surface excess of water to be zero and the activity of component $i$ is written to be the same as that of the bulk fraction. Since we are also relying on behaviour within each binary system to describe the mixture then this parameter can be assumed to be implicitly included in derived surface tension parameters as discussed shortly. Firstly, converting the binary surface tension equation into a differential assuming that the activity $a_{i}$ is represented by the solute mole fraction $x_{i}$ (Eq. 4):

$$
\frac{d \sigma}{d x_{i \mathrm{~B}}}=-R T \Gamma_{i}^{\mathrm{wo}}\left(\frac{K_{i}}{1+K_{i} x_{i \mathrm{~B}}}\right)
$$

Substituting back into Eq. (10):

$n_{\mathrm{is}} \frac{d \ln \left(x_{i \mathrm{~B}} \gamma_{i \mathrm{~B}}\right)}{d x_{i \mathrm{~B}}}+\frac{A}{R T}\left(-R T \Gamma_{i}^{\mathrm{wo}}\left(\frac{K_{i}}{1+K_{i} x_{i \mathrm{~B}}}\right)\right)=0$

Now re-expressing the derivative of $\ln \left(x_{i \mathrm{~B}} \gamma_{i \mathrm{~B}}\right)$ :

$$
\begin{array}{r}
\frac{d \ln \left(x_{i \mathrm{~B}} \gamma_{i \mathrm{~B}}\right)}{d x_{i \mathrm{~B}}}=\frac{d \ln \left(x_{i \mathrm{~B}} \gamma_{i \mathrm{~B}}\right)}{d\left(x_{i \mathrm{~B}} \gamma_{i \mathrm{~B}}\right)} \frac{d\left(x_{i \mathrm{~B}} \gamma_{i \mathrm{~B}}\right)}{d x_{i \mathrm{~B}}} \\
\frac{d \ln \left(x_{i \mathrm{~B}} \gamma_{i \mathrm{~B}}\right)}{d x_{i \mathrm{~B}}}=\frac{1}{x_{i \mathrm{~B}} \gamma_{i \mathrm{~B}}}\left[\gamma_{i \mathrm{~B}}+x_{i \mathrm{~B}} \frac{d \gamma_{i \mathrm{~B}}}{d x_{i \mathrm{~B}}}\right] \\
\frac{d \ln \left(x_{i \mathrm{~B}} \gamma_{i \mathrm{~B}}\right)}{d x_{i \mathrm{~B}}}=\left[\frac{1}{x_{i \mathrm{~B}}}+\frac{1}{\gamma_{i \mathrm{~B}}} \frac{d \gamma_{i \mathrm{~B}}}{d x_{i \mathrm{~B}}}\right]
\end{array}
$$

In line with the assumption that the activity $a_{i}$ is represented by the solute mole fraction $x_{i}$, it is possible to neglect the derivative of activity coefficients since the use of empirical surface tension parameters enables this variation to be included implicitly within the derived values of $\Gamma_{i}^{\text {wo }}$ and $K_{i}$. From this point onward we therefore refer to these variables as $\Gamma_{i}^{\text {wo' }}$ and $K_{i}^{\prime}$. Using this approach, the equation now reduces to:

$\frac{d \ln \left(x_{i \mathrm{~B}} \gamma_{i \mathrm{~B}}\right)}{d x_{i \mathrm{~B}}}=\frac{1}{x_{i \mathrm{~B}}}$

We can now substitute this into Eq. (12) to arrive at the expression:

$n_{\mathrm{is}} \frac{1}{x_{i \mathrm{~B}}}=A \Gamma_{i}^{\mathrm{wo}}\left(\frac{K_{i^{\prime}}}{1+K_{i^{\prime}} x_{i \mathrm{~B}}}\right)$

We can easily derive an expression for $n_{\text {is }}$ in terms of $x_{i \mathrm{~B}}$ by relying on the fact that we are working with each binary system to describe the mixture as manifest in the expression for $\sigma_{\mathrm{ws}}$.

$n_{\text {is }}=n_{i \mathrm{~T}}-n_{i \mathrm{~B}}$

Assuming that the surface excess of water is zero in the binary mixture, we can then write:

$n_{\mathrm{is}}=n_{i \mathrm{~T}}-\frac{x_{i \mathrm{~B}}}{1-x_{i \mathrm{~B}}} n_{\mathrm{wT}}$ 
Substituting this expression into Eq. (17):

$$
\begin{array}{r}
{\left[n_{i \mathrm{~T}}-\frac{x_{i \mathrm{~B}}}{1-x_{i \mathrm{~B}}} n_{\mathrm{wT}}\right] \frac{1}{x_{i \mathrm{~B}}}=A \Gamma_{i}^{\mathrm{wo}}\left(\frac{K_{i}^{\prime}}{1+K_{i}^{\prime} x_{i \mathrm{~B}}}\right)} \\
{\left[n_{i \mathrm{~T}}-\frac{x_{i \mathrm{~B}}}{1-x_{i \mathrm{~B}}} n_{\mathrm{wT}}\right]=x_{i \mathrm{~B}} A \Gamma_{i}^{\mathrm{wo}^{\prime}}\left(\frac{K_{i}^{\prime}}{1+K_{i}^{\prime} x_{i \mathrm{~B}}}\right)} \\
n_{i \mathrm{~T}}-\frac{x_{i \mathrm{~B}}}{1-x_{i \mathrm{~B}}} n_{\mathrm{wT}}=\left(\frac{x_{i \mathrm{~B}} A \Gamma_{i}^{\mathrm{wo}} K_{i}^{\prime}}{1+K_{i}^{\prime} x_{i \mathrm{~B}}}\right)
\end{array}
$$

Now deriving an expression for $x_{i \mathrm{~B}}$ :

$$
\begin{aligned}
& x_{i \mathrm{~B}}^{2}\left(A \Gamma_{i}^{\mathrm{wo}^{\prime}} K_{i}^{\prime}-n_{\mathrm{wT}} K_{i}^{\prime}-n_{i \mathrm{~T}} K_{i}^{\prime}\right) \\
& +x_{i \mathrm{~B}}\left(n_{i \mathrm{~T}} K_{i}^{\prime}-n_{i \mathrm{~T}}-n_{\mathrm{wT}}-A \Gamma_{i}^{\mathrm{wo}{ }^{\prime}} K_{i}^{\prime}\right)+n_{i \mathrm{~T}}=0
\end{aligned}
$$

Thus we have a quadratic expression for $x_{i \mathrm{~B}}$. A solution to a basic quadratic equation written as:

$x_{i \mathrm{~B}}^{2} a+x_{i \mathrm{~B}} b+c=0$

Is simply given as:

$x_{i \mathrm{~B}}=\frac{-b \pm \sqrt{b^{2}-4 a c}}{2 a}$

Where the negative root must be used to obtain mole fractions between 0 and 1 . To solve the above we need to calculate concentrations $n_{i \mathrm{~T}}$ and $n_{\mathrm{wT}}$. The total wet volume $\left(\mathrm{m}^{3}\right)$ is given as:

$V_{\text {drop }}=V_{\mathrm{w}}+V_{i}=n_{\mathrm{wT}} v_{\mathrm{w}}+n_{i \mathrm{~T}} v_{i}$

Where:

$v_{\mathrm{w}}=1.8 \times 10^{-5} \mathrm{~m}^{3} \mathrm{~mol}^{-1}$

$v_{i}=\frac{M_{i}}{\rho_{i}}$

$n_{i \mathrm{~T}}=\frac{V_{\mathrm{dry}} \rho_{i}}{M_{i}}$

and therefore:

$n_{\mathrm{wT}}=\frac{\left(V_{\mathrm{drop}}-n_{i \mathrm{~T}} v_{i}\right)}{v_{\mathrm{w}}}$

\section{Application}

\subsection{Binary mixtures}

It is useful to perform an example of a binary system using the above formulation. Take a hypothetical compound for which the parameters $K_{i}^{\prime}$ and $\Gamma_{i}^{\text {wo' }}$ were chosen arbitrarily to be 400 and 0.005 , respectively. Take a
$10 \mathrm{~nm}$ dry radius aerosol at a wet size of $100 \mathrm{~nm}$. We can prescribe the following parameters $M_{i}=200 \mathrm{~g} \mathrm{~mol}^{-1}$; $\rho_{i}=1500 \mathrm{~kg} \mathrm{~mol}^{-1}$ such that we get the following values: $\quad v_{i}=1.33^{10}-4 \mathrm{~m}^{3} \mathrm{~mol}^{-1} ; \quad n_{i \mathrm{~T}}=3.141610^{-20} \mathrm{~mol}$; $n_{\mathrm{wT}}=2.324810^{-16} \mathrm{~mol} ; A=1.256610^{-13} \mathrm{~m}^{2}$. Then we can calculate values for the original bulk mole fraction $x_{i \mathrm{~B}}^{o}$ and that after accounting for bulk to surface partitioning. Only $50 \%$ of material remains in the bulk phase after partitioning. If we change the wet size to 50,200 and $500 \mathrm{~nm}$ the ammount of material remaining in the bulk changes to: 35,65 and 82 perent. Thus, as the surface to volume ratio decreases, the ammount of material partitioned to a surface layer, relative to the original bulk value, decreases. This is to be expected. Similarly, the effect is expected to reduce with increasing dry size. If we increase the dry size to $100 \mathrm{~nm}$ and use the same wet/dry ratios as the latter example, the percentage of material remaining in the bulk after partitioning is predicted to be: 86,95 and 98 accordingly. These values are greater than the $10 \mathrm{~nm}$ example, suggesting bulk/surface partitioning reduces.

Unfortunately, a extensive database of empirically determined values of $K_{i}$ and $\Gamma_{i}$ does not exist. However, we can initially prescribe values according to observed surface tension isotherms, such as those presented by Topping et al. (2007) and Booth et al. (2009). In Fig. 1a, a collection of isotherms informed from pendant drop measurements, as reported by Topping et al. (2007) and Booth et al. (2009) are given. Figure $1 \mathrm{~b}$ plots the ratio of bulk concentrations after consideration of partitioning to those without, for a broad range of surface tension parameters. In this example the dry and wet sizes were arbitrarily chosen to be $10 \mathrm{~nm}$ and $200 \mathrm{~nm}$. Placed on the same graph are points highlighting the same compounds presented in Fig. 1a. For example, the removal of material from the bulk to surface layer is much more pronounced for cis-pinonic acid relative to malonic acid. One would expect this by simple analysis of the isotherm presented in Fig. 1a. It must be noted that the same values $M_{i}=200 \mathrm{~g} \mathrm{~mol}^{-1} ; \rho_{i}=1500 \mathrm{~kg} \mathrm{~mol}^{-1}$ as stated in the previous example have been used for purely illustrative purposes.

\subsection{Ternary and higher order mixtures}

The equations presented in the previous section can be applied to mixtures of any number of components as dictated by the surface tension model on which they are based. It is important to remember that doing so assumes the partitioning behaviour within a binary aqueous solution pertains in the mixture, with the influence of dilution accounted for by using the total concentration of water in Eq. (23). A brief discussion of the impact of neglecting the effect of common ions in inorganic/organic mixtures, where the presence of sodium chloride can lower the surface tension of a mixed sodium chloride-sodium dodecylsuplhate particle, is considered by comparison with output from a more detailed ternary 
Table 1. Compounds and properties used within a multicomponent example. Data derived from Topping et al. (2007) and Booth et al. (2009).

\begin{tabular}{cccccc}
\hline Compound & $\Gamma_{i}^{\text {wo }}(\mathrm{mmol} / \mathrm{m} 2)$ & $K_{i}^{\prime}$ & $M_{i}(\mathrm{~kg})$ & $\rho_{i}(\mathrm{~kg} / \mathrm{m} 3)$ & $N_{i}$ \\
\hline Malic & 0.0058 & 22.80 & $134.09 \times 10^{-3}$ & 1609 & 1 \\
cis-Pinonic & 0.0026 & $2.62 \times 10^{4}$ & $184.23 \times 10^{-3}$ & 786 & 1 \\
Malonic & 0.0033 & 57.65 & $104.06 \times 10^{-3}$ & 1630 & 1 \\
Glutaric & 0.0005 & 1045.94 & $132.11 \times 10^{-3}$ & 1316 & 1 \\
SR Fulvic acid & 0.0025 & 35942.03 & $732.59 \times 10^{-3}$ & 1400 & 1 \\
\hline
\end{tabular}

Table 2. Critical supersaturations for Köhler curves calculated with different mass fractions of SDS and NaCl. Approach 1 shows predictions using equations presented in this study. Approach 2 are the results of Sorjamaa et al. (2004). Approaches 1 and 2 consider combined effects on the Raoult and Kelvin terms. Approach 3 are the results of Li et al. (1998) who only considers the impact on surface tensions

\begin{tabular}{cccc}
\hline SDS mass fraction & Approach 1 Sc(\%) & Approach 2 Sc(\%) & Approach 3 Sc(\%) \\
\hline 0 & 0.103 & 0.103 & 0.103 \\
0.2 & 0.123 & 0.123 & 0.121 \\
0.5 & 0.177 & 0.167 & 0.157 \\
0.8 & 0.292 & 0.256 & 0.214 \\
1.0 & 0.305 & 0.304 & 0.247 \\
\hline
\end{tabular}

model. Before this, the following examples illustrate how the equations are applied to a multi-component mixture of any number of compounds. For example, take an aerosol comprised of 5 compounds presented in Table 1.

Given the relative molar abundance of each compound $N_{i}$ and individual densities $\rho_{i}$, thus molar volumes, it is possible to calculate the total number of moles, $n_{i \mathrm{~T}}$, of each compound for a give dry size. For example, if we have each compound in an equimolar mixture (such that the relative molar abundance of each compound =1), using ideal mixing we know that the volume of the dry particle is a sum of the individual molar volumes of the contributing compounds:

$V_{\mathrm{dry}}=\sum V_{i}=\sum n_{i \mathrm{~T}} v_{i}$

This can be written similarly as:

$V_{\mathrm{dry}}=\sum V_{i}=\left(\sum N_{i \mathrm{~T}} v_{i}\right) \times \beta$

Where $\beta$ is a conversion factor to obtain $n_{i}$ from $N_{i}$ such that $n_{i \mathrm{~T}}=N_{i} \times \beta$. On obtaining values for $n_{i \mathrm{~T}}$ one can proceed via Eqs. (23-30) to obtain values for $x_{i \mathrm{~B}}$. Keeping the same compounds listed in Table 1, Fig. 2a plots calculated bulk/surface concentration ratios as a function of wet droplet size for a $50 \mathrm{~nm}$ dry radius particle. It is interesting to note that this ratio is particular low for cis-pinonic acid and SR Fulvic acid, which is to be expected based on their ability to lower surface tensions in aqueous solutions. In Fig. $2 b$ the predicted critical saturation ratio is shown for different permutations of bulk/surface partitioning within Köhler theory for the same 5 component mixture. In the legend: $a w_{\text {Nopartitioning }} \sigma_{W S \text { Nopartitioning }}$ refers to the "traditional" use of Köhler theory in which the total concentrations of each compound define the Raoult and Kelvin terms, $a w_{\text {Nopartitioning }} \sigma_{W}$ Spartitioning refer to bulk/surface partitioning considerations only affecting the surface tension term within the kelvin equation (e.g. Li et al., 1998), $a w_{\text {Partitioning }} \sigma_{\text {Partitioning }}$ refer to bulk/surface partitioning considerations affecting both the surface tension term within the kelvin equation and the Raoult term (e.g. Sorjamaa 2004 and this study), $a w_{\text {NoPartitioning }} \sigma_{\mathrm{w}}$ refers to the "traditional" use of Köhler theory in which the total concentrations of each compound define the Raoult terms and the surface tension is assumed to be that of pure water. The figure shows that a "complete" consideration of bulk/surface partitioning in this case results in predicted critical saturation ratios close to and slighty above the traditional approach in which the surface tension of water is used. Conversely, the traditional approach of using total concentrations to define both the Raoult and Kelvin terms in the Köhler equation produces significantly lower critical points. It should be noted here that ideality was assumed when constructing the Köhler curve.

As stated previously, the approach presented here neglects some phenomena postulated to occur at a bulk/surface interface. For example, the surface tension framework described in the previous section can be modified to account for competing adsorption ( $\mathrm{Li}$ and $\mathrm{Lu}, 2001$ ). This will form the focus of another publication. However, comparison with bulk data by Topping et al. (2007) and more recently, Booth et al. (2009), could not conclusively determine whether this increased level of complexity is necessary. Another neglected effect is the impact of inorganic components on the critical miscelle concentration. Under specific conditions it may be 
(a)
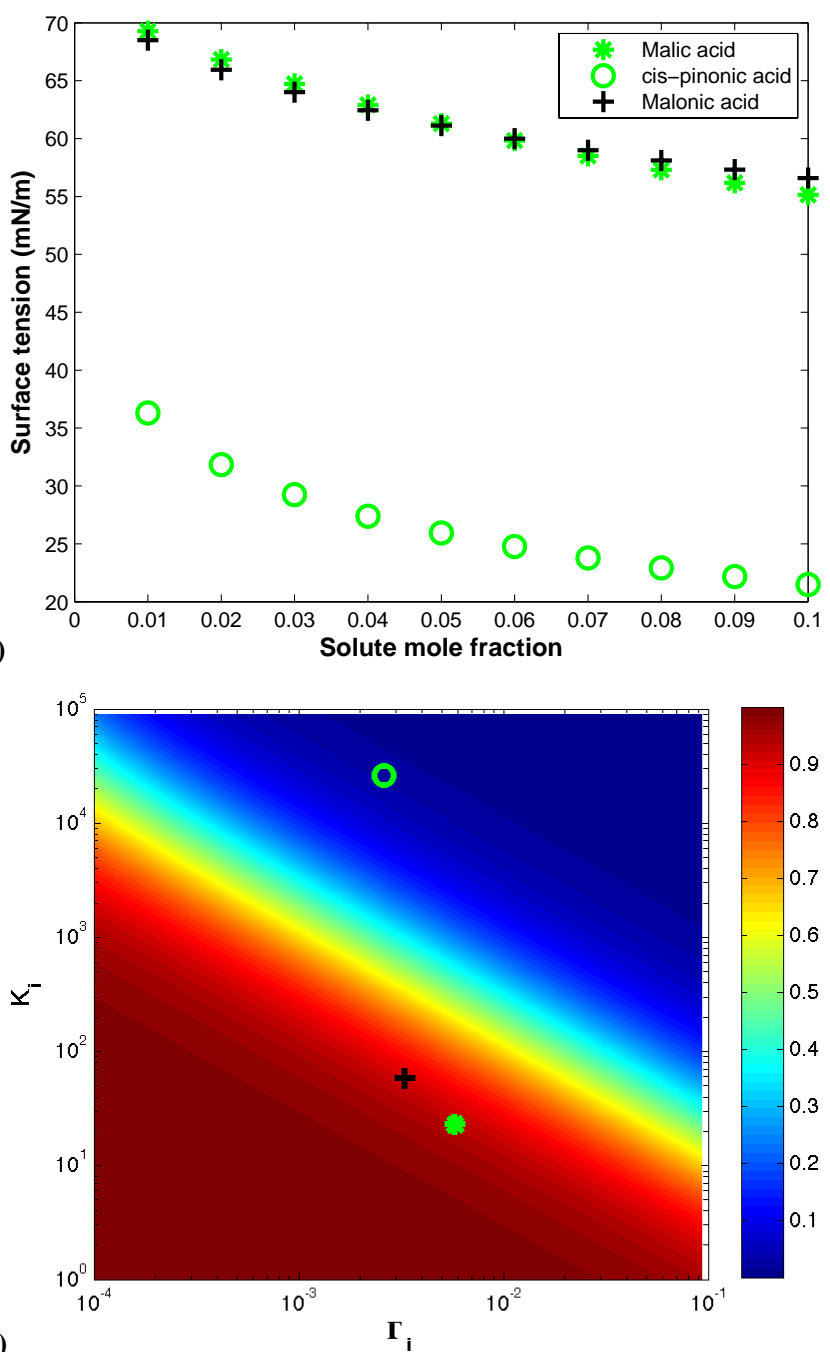
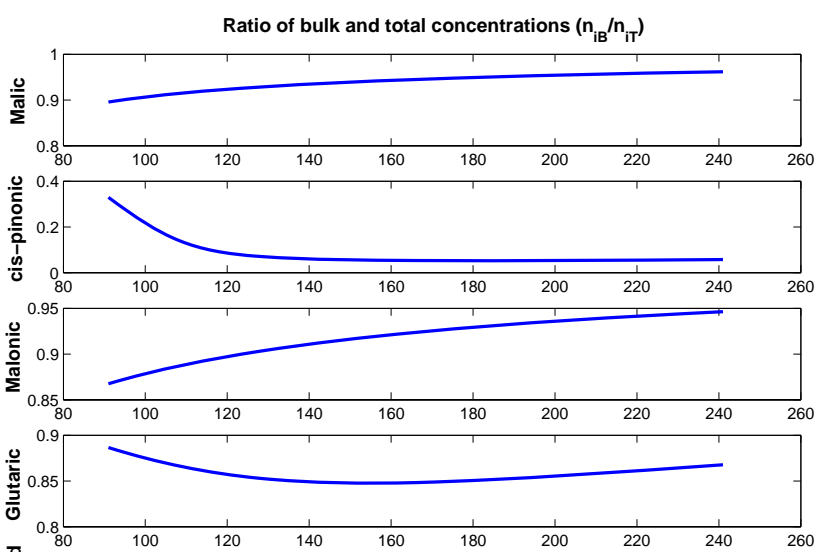

(a)
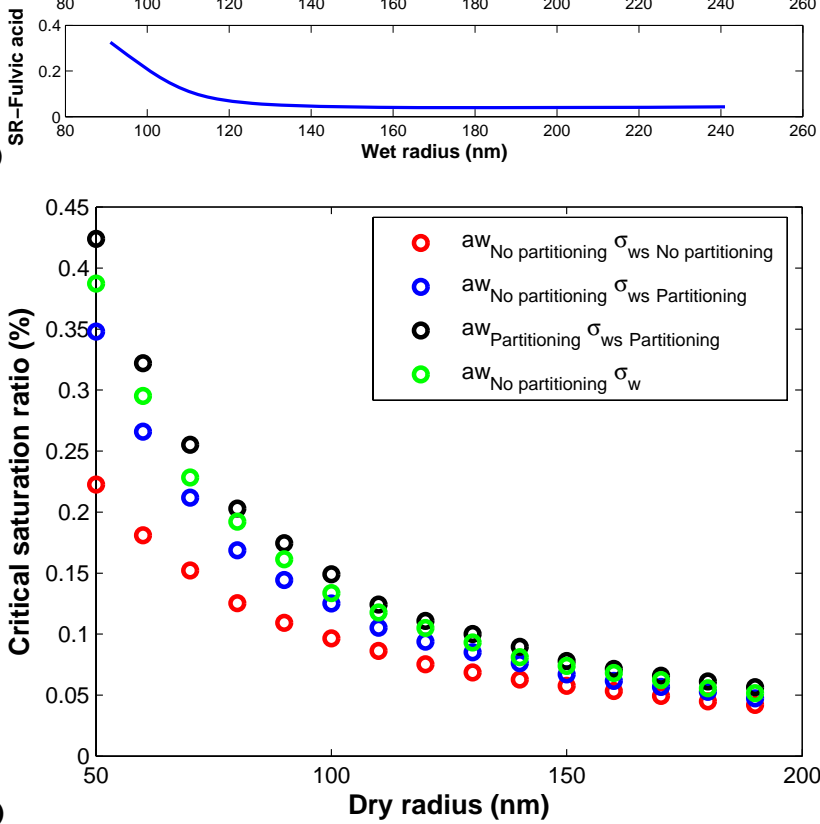

Fig. 1. (a) Surface tension isotherms. (b) Ratio of bulk concentrations after partitioning to those without.

appropriate to consider the partitioning into miscelles, using boundary conditions similar to the inclusion of solubility (e.g. Shulman et al., 1996). In the detailed frameworks presented by Sorjamaa et al. (2004) and Raatikainen and Laaksonen (2010), the effect of common ions are analysed. For example, whilst added sodium chloride increases surface tension of pure water, it decreases surface tension of aqueous sodium dodecyl sulphate (SDS) solution (e.g. Li et al., 1998). Since the "pivot" assumption used to arrive at the analytical expressions presented in this paper is that the partitioning behaviour within a binary aqueous solution pertains in the mixture, with the influence of dilution accounted for by using the total concentration of water, it is possible and useful to compare predictions between both approaches. Table 2 shows predicted critical saturation ratios for mixtures of SDS-NaCl at varying mass fractions.
Fig. 2. (a) Ratio of bulk and total concentrations as a function of wet droplet size for a dry $50 \mathrm{~nm}$ aerosol. (b) Critical saturation ratio as a function of dry size for different model permutations. See Sect. 3.2 in main text for a more detailed discussion.

The first column presents the results of Sorjamaa et al. (2004) who explicitly combined the effects of non-ideality in the partitioning framework and a surface tension parameterisation based on measurements of each mixture, on the Raoult and kelvin term in the Köhler equation. The second column presents the results of $\mathrm{Li}$ et al. (1998) who considered only the effect of partitioning on the surface tension of the solution, using the same parameterisation of Sorjamaa et al. (2004). The third column presents results from the equations presented in this publication, assuming the surface tension of the mixture can be described using the model of $\mathrm{Li}$ and $\mathrm{Lu}$ (2001). In all cases, the osmotic coefficients and dissociation factors for both $\mathrm{NaCl}$ and SDS are the same as those presented by Sorjamaa et al. (2004). For a binary 
SDS/water system the approach presented here can replicate the predicted critical point as presented by Sorjamaa et al. (2004) within $0.001 \%$. With an $80 \%$ mass ratio of SDS within the multicomponent system, this study gives a critical saturation ratio which is higher by 0.036 , compared to a difference of -0.042 compared with the Li et al. (1998) model. At a $50 \%$ mass ratio, the difference reduces to 0.01 , the two models equal to within 3 decimal places at a $20 \%$ mass ratio. Thus, there is evidently a difference in approaches when the common ion effect is present. This is to be expected given the differing levels of complexity in both appraoches and can be partly expalined by the different surface tension model employed. Specifically, as already stated, the $\mathrm{Li}$ and $\mathrm{Lu}$ (2001) model assumes the binary surface tensions can be employed to describe the behaviour of the mixture. In this system, it is know that $\mathrm{NaCl}$ decreases surface tension of aqueous sodium dodecyl sulphate (SDS) solution. Thus the use of the $\mathrm{Li}$ and $\mathrm{Lu}$ (2001) model tends to over-predict the final surface tension even after partitioning has been taken into account, resulting in an additional forcing which increases the final critical saturation ratio. It is possible to include a seperate empirically derived surface tension fit to calculate the equilibrium saturation ratio within the Kohler equation for a specific mixture within the framework presented here. However, for illustrative purposes the same model used to define the bulk/surface partitioning equations are used in the results presented in Table 2 for consistency.

It is difficult to extrapolate the deviations found here to other mixtures of atmospherically relevant compounds or multicomponent mixtures of higher order. Given that purely theoretical models of surface tension perform poorly when compared with experimental data (Topping et al., 2007), it is evident that laboratory data is essential for deriving empirical surface tension fits for mixtures, or for input into mixing rules. Indeed, it must be stressed that the level of accuracy required to capture this phenomena for a broad range of organic functionality and complexity must be answered using empirical data on the CCN behaviour of real world aerosol and sensitivity studies within large scale prognostic models.

The equations presented in the recent publication of Raatikainen and Laaksonen (2010) are specifically designed to diagnose specific phenomena occurring at bulk/surface interfaces in, upto, ternary mixtures. As such they are important predictive and diagnostic frameworks. We believe the overall effect of partitioning in mixtures of any number of components can be captured by the framework presented in this report such that the altered critical saturation ratio should be measurable by conventional CCN instruments. However, this, of course, requires testing with appropriate measurements as stated previously. Perhaps more importantly, the impact of the bulk/surface partitioning effect on cloud properties should be assessed within a large scale modelling framework, giving insight into the level of detail required.

\subsection{Computational performance}

It is useful to briefly compare the computational cost of using the analytical expression with regards to an iterative method. For any given set of surface tension parameters only 23 floating point operations, or FLOPS, are required. The number of corresponding FLOPS required in the iterative method depends on the efficiency of root finding scheme. For example, applying a newton-raphson search with root bracketing on the cis-pinonic acid example presented in Fig. 1 requires 498 FLOPS. Similarly, the malonic acid example requires 423 FLOPS. These values represent binary systems using the same assumptions employed in the analytical method. Using the coupled method presented by Sorjamaa et al. (2004), accounting for activity coefficient variation with 5th order polynomials (including the derivative), requires 933 FLOPS. These values were simply derived by assuming a root finding tolerance of $<0.1 \%$ change in the bulk concentration. For multi-component systems the cost of the analytical expression can be extrapolated linearly. For example, for a 10 component testcase, the analytical solution requires 230 FLOPS, whereas the iterative search employing the same assumptions may take 4230-4980 FLOPS for similar compounds. The coupled approach would require a considerable amount more, though it is difficult to quantify since it is presently not possible to account for more than 3 compounds.

\section{Conclusions and future work}

An analytical solution to calculate the removal of material from a bulk to a surface layer has been derived using a well established and validated surface tension model. The potential applicability to multi-component mixtures requires only surface tension parameters from binary systems. Whilst assumptions regarding behaviour at the surface layer have been made to facilitate derivation, we feel the framework presented can capture the overall impact of bulk-surface partitioning. This requires validating against measured $\mathrm{CCN}$ data of known composition from laboratory studies. However, it is also important to apply the framework to ensemble mixtures from smog chamber and ambient studies where possible. The computational cost of using the solution presented in this paper is roughly a factor of 20 less than a similar iterative approach.

We will revisit the derivation using surface tension models that account for competing adsorption at the surface layer as described by Topping et al. (2007). Perhaps more importantly, we also intend to derive a generalised predictive framework in which the surface tension parameters $K_{i}$ and $\Gamma_{i}$ can be linked to broad functional groups and characteristics of the individual organic compounds. This requires compilation of an extensive database of binary systems. This will form the focus of a future publication and will allow sensitivity studies to be carried out along the lines of those presented 
by McFiggans et al. (2010). It must be stressed that the level of accuracy required to capture this phenomena for a broad range of organic functionality and complexity must be answered using empirical data on the CCN behaviour of real world aerosol and sensitivity studies within large scale prognostic models.

Acknowledgements. D. Topping is currently funded by NCAS. The author would like to thank Harri Kokkola, Tomi Raatikainen and Sami Romakkaniemi for their fruitful discussions on the issue addressed within the paper.

Edited by: R. Sander

\section{References}

Booth, A. M., Topping, D. O., McFiggans, G., and Percival, C. J.: Simple model for prediction of surface tension of mixed surfactant solutions, PCCP., 11(36), 8021-8028, 2009.

Fainerman, V. B., Miller, R., and Aksenenko, E. V.: Simple model for prediction of surface tension of mixed surfactant solutions, Adv. Col. Int. Sci., 96(1-3), 339-359, 2002.

Fainerman, V. B. and Miller, R.: Simple method to Estimate Surface tension of Mixed Surfactant Solutions, J. Phys. Chem. B, 105, 11432-11438, 2001a.

Fainerman, V. B., Wustneck, R., and Miller, R.: Surface tension of mixed surfactant solutions, Tenside Surfact Det., 38(4), 224-229, $2001 b$.

Hallquist, M., Wenger, J. C., Baltensperger, U., Rudich, Y., Simpson, D., Claeys, M., Dommen, J., Donahue, N. M., George, C., Goldstein, A. H., Hamilton, J. F., Herrmann, H., Hoffmann, T., Iinuma, Y., Jang, M., Jenkin, M. E., Jimenez, J. L., Kiendler-Scharr, A., Maenhaut, W., McFiggans, G., Mentel, Th. F., Monod, A., Prévôt, A. S. H., Seinfeld, J. H., Surratt, J. D., Szmigielski, R., and Wildt, J.: The formation, properties and impact of secondary organic aerosol: current and emerging issues, Atmos. Chem. Phys., 9, 5155-5236, doi:10.5194/acp-95155-2009, 2009.

Hu, Y. F. and Lee, H.: Prediction of the surface tension of mixed electrolyte solutions based on the equation of Patwardhan and Kumar and the fundamental Butler equations, J. Col. Int. Sci., 269(2), 442-448, 2004.
Kokkola, H., Sorjamaa, R., Peraniemi, A., Raatikainen, T., and Laaksonen, A.: Cloud formation of particles containing humic-like substances, Geophys. Res. Lett., 33, L10816, doi:10.1029/2006GL026107, 2006.

Laaksonen, A., McGraw, R., and Vehkamaki, H.: Liquid-drop formalism and free-energy surfaces in binary homogeneous nucleation theory, J. Chem. Phys., 111, 2019-2027, 1999.

Li, Z., Williams, A. L., and Rood, M. J.: Influence of soluble surfactant properties on the activation of aerosol particles containing inorganic solute, J. Atmos. Sci., 55, 1859-1866, 1998.

Li, Z. B. and Lu, B. C. Y.: Surface tension of aqueous electrolyte solutions at high concentrations representation and prediction, Chem. Eng. Sci., 56(8), 2879-2888, 2001.

McFiggans, G., Topping, D., and Barley, M.: The sensitivity of Secondary Organic Aerosol component partitioning to the predictions of component properties: part 1; a systematic evaluation of available predictive techniques, accepted, Atmos. Chem. Phys. Discuss., 2010.

Raatikainen, T. and Laaksonen, A.: A simplified treatment of surfactant effects on cloud drop activation, Geosci. Model Dev. Discuss., 3, 1139-1159, doi:10.5194/gmdd-3-1139-2010, 2010.

Shulman, M. L., Jacobson, M. C., Charlson, R. J., Synovec, R. E., and Young, T. E.: Dissolution behavior and surface tension effects of organic compounds in nucleating cloud drops, Geophys. Res. Lett., 23, 277-280, 1996.

Sorjamaa, R., Svenningsson, B., Raatikainen, T., Henning, S., Bilde, M., and Laaksonen, A.: The role of surfactants in Köhler theory reconsidered, Atmos. Chem. Phys., 4, 2107-2117, doi:10.5194/acp-4-2107-2004, 2004.

Topping, D. O., McFiggans, G. B., and Coe, H.: A curved multi-component aerosol hygroscopicity model framework: 2Including organics, Atmos. Chem. Phys., 5, 1223-1242, 2005, http://www.atmos-chem-phys.net/5/1223/2005/.

Topping, D. O., McFiggans, G. B., Kiss, G., Varga, Z., Facchini, M. C., Decesari, S., and Mircea, M.: Surface tensions of multicomponent mixed inorganic/organic aqueous systems of atmospheric significance: measurements, model predictions and importance for cloud activation predictions, Atmos. Chem. Phys., 7, 2371-2398, doi:10.5194/acp-7-2371-2007, 2007. 